\title{
Capital humano e innovación en el proceso de integración del aprovechamiento forestal maderable en el ejido Gómez Tepeteno, Tlatlauquitepec, Puebla
}

\section{Human capital and innovation in the integration process of logging in the ejido Gómez Tepeteno, Tlatlauquitepec, Puebla}

\author{
Diana Lorena Sánchez-Vidañal, Esteban Valtierra-Pacheco ${ }^{*}$ \\ Manuel de Jesús González-Guillén² y Aurelio León-Merino'
}

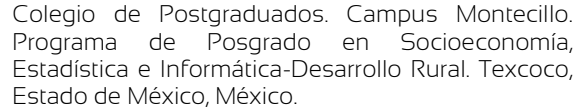

Colegio de Postgraduados. Campus Montecillo. Programa de Posgrado en Socioeconomía, Estadística e Informática-Desarrollo Rural. Texcoco, Estado de México, México.

\author{
Colegio de Postgraduados. Campus Montecillo. \\ Programa de Posgrado en Ciencias Forestales. \\ Texcoco, Estado de México, México.
}

\section{RESUMEN}

El ejido Gómez Tepeteno tomó control del uso y manejo de sus recursos forestales maderables y no maderables en la década de 1970. Uno de los procesos más importantes al que ha tenido que poner atención es el desarrollo de capacidades del personal que participa en el aprovechamiento e industrialización de la madera y, más recientemente, en la adopción de procesos de innovación tecnológica. El objetivo del presente estudio fue analizar el proceso de innovación y formación de capital humano en la integración de la cadena de valor maderable del ejido. La investigación se realizó a través de entrevistas semiestructuradas dirigidas a actores clave, encuestas estructuradas a trabajadores de la industria forestal y observación directa de todas las fases productivas. Los resultados muestran que el ejido ha hecho avances importantes en el desarrollo del capital humano en las innovaciones siguientes: 1) uso de la forcípula electrónica para el cálculo de la cubicación y la documentación de madera en rollo, 2) aplicación de un sistema de control contable y financiero, 3) producción de madera estufada, tanto de madera aserrada propia como de maquila y 4) integración de un modelo de cadena de valor maderable. El ejido Gómez Tepeteno ha entendido que para el mejor aprovechamiento maderable debe generar sistemas permanentes de innovación tecnológica de procesos de corte e industrialización que implican inversión y capitalización en el corto, mediano y largo plazos.

PALABRAS CLAVE: adopción de tecnología, capital natural, desarrollo de capacidades, forcípula, madera estufada, organización social.

\section{ABSTRACT}

The ejido Gomez Tepeteno took control of the use and management of their wood and non-wood forest resources in the 1970s. One of the most important processes they paid attention to was the development of staff capacity-building involved in logging and industrialization and, more recently, into the adoption of technological innovation processes. The objective of this study was to analyze the innovation process and human capital building in the integration of the timber forest chain in this ejido. The research was carried out using semi-structured interviews targeted to key actors, structured surveys to forest industry workers, and direct observation on all the productive phases. The results show that the ejido has made important achievements on human capital development on the following innovations: 1) the use of automatic data caliper to cubage and documentation of roundwood, 2) implementation of an accounting and 
financial control system, 3) production of kiln-dried wood from sawnwood of its own or processed for others, and 4) integration of a value network model for wood products. The ejido Gómez Tepeteno has understood that to improve wood production, they must generate permanent systems of technological innovations on logging and transformation processes that involve investment and capitalization in short, medium and long-terms.

KEYWORDS: adoption of technology, natural capital, capacity building, automatic data caliper, kiln-dried wood, social organization.

\section{INTRODUCCIÓN}

Los bosques cubren $31 \%$ del área total de la tierra (Organización de las Naciones Unidas para la Alimentación y la Agricultura [FAO], 2010) y desempeñan un papel primordial desde el punto de vista social, económico y ambiental. Las áreas forestales de México abarcan 71\% de su territorio (Secretaría de Medio Ambiente y Recursos Naturales [Semarnat], 2012), de las cuales $80 \%$ son de propiedad social (FAO, 2004) y están habitadas por aproximadamente 11 millones de personas equivalente a 9.5\% de la población total del país (Semarnat, 2014).

Los recursos forestales son un capital natural, que bien manejados contribuyen a satisfacer necesidades básicas de la población, ingresos y mejora de la calidad de vida de comunidades indígenas y rurales (López-Barrera, Velázquez y Merino-Pérez, 2010). Estas comunidades han gestionado y aprovechado sus recursos naturales basadas en conocimientos, prácticas, reglas y creencias tradicionales (Chao, 2012). Sin embargo, las comunidades forestales presentan los niveles más altos de marginación y pobreza en el país (Semarnat, 2014), mostrando grados de desarrollo muy precarios.

Boisier (2003) indica que el desarrollo es el resultado de la interacción y las relaciones entre varios factores y procesos con el entorno, tales como las relaciones de confianza, el papel de las instituciones y la socialización del conocimiento en una comunidad. Existen cuatro dimensiones que influyen en la decisión de comunidades y ejidos para organizarse y hacer uso de sus recursos: la dimensión social, la ambiental, la económica y la políticoinstitucional. Esta última jugó un papel determinante en la historia del sector forestal en México cuando la explotación de los recursos forestales estaba a cargo de concesionarios industriales (papeleras y madereras). Esta etapa abarcó desde la década de 1940 (Merino-Pérez y SeguraWarnholtz, 2007), prolongándose por más de 40 años, hasta que en 1986 se emitió el decreto de la Ley Forestal (H. Congreso de la Unión, 1986), que finalizó las concesiones forestales en México. Fue así que los campesinos apoyados por funcionarios públicos y profesionales forestales retomaron el control sobre el uso y manejo de sus recursos forestales y se promovió la creación de empresas forestales comunitarias (EFC) (Bray y Merino-Pérez, 2004).

Una EFC tiene su origen y pertenece a una comunidad agraria o ejido y opera a través de miembros electos mediante asambleas (Bray y Merino-Pérez, 2004). TorresRojo, Guevara-Sanginés y Bray (2007) añaden que la lógica, objetivos, administración y distribución de beneficios de este tipo de empresas las diferencian de aquellas que operan con capital privado. Gasca-Zamora (2014) explica que la racionalidad de las EFC promueve la distribución de ganancias y somete a consideración de la asamblea la inversión de las mismas en obras y servicios en beneficio de la comunidad (caminos, agua, luz, hospitales, escuelas, entre otras).

A pesar de sus particularidades, y al estar inmersas en un contexto económico global, las EFC compiten para sobrevivir pues son tratadas con criterios de mercado igualmente estrictos como cualquier otra empresa (TorresRojo et al., 2007). Por lo que estas deben transitar de un modelo de producción tradicional de cadenas productivas a la formación de cadenas de valor. Una cadena de valor pone en el centro de sus decisiones al consumidor, y la producción se enfoca a satisfacer demandas específicas y opera bajo la premisa ganar-ganar, por tanto, la relación 
empresa, consumidor y proveedores es estrecha (Bovet y Joseph, 2000).

Uno de los elementos centrales de las EFC es la formación y desarrollo del capital social. El capital social es concebido como la capacidad de las personas para trabajar en grupo, para cooperar entre sí y lograr objetivos comunes sobre la base de un conjunto de normas y valores compartidos (Fukuyama, 2003).

Las primeras EFC autónomas que se intentaron crear en México fueron en regiones que habían estado sujetas a vedas forestales, por ejemplo, en Chignahuapan, Puebla; Zacualtipán, Hidalgo; Huayacocotla, Zongolica y Cofre de Perote en Veracruz; Tlaxco, Tlaxcala; y Valle de Bravo en el Estado de México (Bray et al., 2003). El desarrollo de las EFC estuvo limitado por la escasez de capital humano, tecnología y por la presencia de intermediarios (contratistas) en la comercialización de la madera (MerinoPérez y Segura-Warnholtz, 2007), por lo cual la Ley Forestal de 1986 (H. Congreso de la Unión, 1986) estableció: "Promover la educación, la capacitación y la investigación en materia forestal, para satisfacer las necesidades de recursos humanos y de tecnología adecuada al pais" (H. Congreso de la Unión, 1986).

En años posteriores, y una vez creada la Secretaría de Medio Ambiente y Recursos Naturales (Semarnat) y la Comisión Nacional Forestal (Conafor), este mismo propósito fue establecido en la Ley General de Desarrollo Forestal Sustentable vigente (LGDFS), cuyo artículo 3, Fracción XXXII, establece como objetivo específico: "Fomentar la cultura, educación, capacitación, investigación y desarrollo tecnológico forestal" (H. Congreso de la Unión, 2003).

En este sentido, el manejo forestal comunitario, además de contribuir al capital social, ha sumado a la formación de capital bumano (Chapela, 2007) como elementos fundamentales para el aprovechamiento forestal sostenible. La teoría del capital bumano sostiene que es producto de una decisión deliberada de inversión. Consiste en las habilidades y conocimientos adquiridos por los individuos a lo largo de su vida. Estos se convierten en herramientas que les permiten participar de forma eficiente en los procesos productivos (Schultz, 1972).
Chapela (2012) señala que entre los principales problemas del sector forestal se encuentran: a) débil inversión en la generación, transferencia y aplicación de conocimientos, b) visión empresarial escasa, c) capacidades administrativas y gerenciales deficientes, d) rezago tecnológico en la industria forestal y e) posibilidades limitadas para la adopción de tecnología, entre otros.

En 2017, la Semarnat, la Conafor y el Programa de las Naciones Unidas para el Desarrollo (PNUD) a través del Fondo para el Medio Ambiente Mundial (GEF por sus siglas en inglés) abordaron los temas relacionados con el capital humano e innovaciones en el proceso de transformación de la materia prima por medio de la presentación de diez casos de éxito. Estos estudios giraron en torno a la diversificación productiva, organización social para la comercialización, participación de los jóvenes en la industria forestal, exportación de madera certificada y modernización industrial. Aun con estas iniciativas, se requiere generar más información y conocimiento sobre los demás eslabones de la cadena productiva forestal.

\section{OBJETIVOS}

El objetivo del presente estudio fue identificar los procesos de formación de capital humano e introducción de innovaciones en el aprovechamiento y transformación forestal maderable en el ejido Gómez Tepeteno, municipio de Tlatlauquitepec, Puebla.

\section{MATERIALES Y MÉTODOS}

Los resultados presentados aquí son parte de una investigación de mayor alcance que se realizó en el ejido Gómez Tepeteno, localizado en la parte sureste del municipio de Tlatlauquitepec, Puebla. Posee una superficie total de 2406.7 ha, de las cuales 1654.2 ha (68.7\% del total) son tierras de uso común. Del total, 1303.0 ha están destinadas a la producción forestal y 718.3 ha son tierras parceladas de uso agrícola.

El enfoque de la investigación fue descriptivo de un estudio de caso. Las técnicas de investigación empleadas fueron: observación, encuesta y entrevistas semiestructuradas. La observación permitió identificar 
procesos, roles, formas de organización y describir actividades. El trabajo de campo se realizó durante mayo y junio de 2017, en este periodo se observaron todas las fases de la cadena productiva: planeación del aprovechamiento forestal, capacitación, ejecución, acompañamiento técnico en el uso de tecnología, transporte, comercialización de materia prima y transformación. Las entrevistas semiestructuradas se aplicaron a 10 actores clave que incluyeron a tres miembros del Comisariado Ejidal, un capacitador en el manejo de la forcípula electrónica, un asesor técnico forestal (ATF), un jefe de monte, un coordinador, una documentadora, un motosierrista principal y un jefe de la industria de transformación. El criterio de selección de los actores clave fue la posición de cargo dentro del aprovechamiento y el grado de experiencia en torno a las innovaciones y la cadena productiva. Con la encuesta se buscó censar a los trabajadores del aserradero y se logró encuestar a 21 de los 24 trabajadores para determinar los procesos de formación de capital humano. Adicionalmente, se utilizaron algunos datos cualitativos de una encuesta aplicada a 48 ejidatarios para enriquecer el presente artículo, los resultados estadísticos de esta encuesta no se muestran porque tenían un objetivo diferente al del presente artículo. La mayor parte de los resultados presentados son cualitativos derivados de las entrevistas semiestructuradas. Los resultados cuantitativos sobre la industria se derivan de la encuesta aplicada a los trabajadores del aserradero.

\section{RESULTADOS Y DISCUSIÓN}

A partir de 1970, el ejido Gómez Tepeteno tomó bajo control la conservación y el aprovechamiento sustentable de sus bosques cuando terminaron las concesiones forestales a las industrias madereras y papeleras. A partir de ese momento inició un largo proceso organizativo para constituir una EFC, que ha sido un proceso de aprendizaje de habilidades y conocimientos y de planeación del manejo forestal de sus bosques, que no ha sido fácil.

Aguirre-Calderón (2015) menciona que el manejo forestal comprende decisiones y actividades encaminadas al aprovechamiento de los recursos forestales de manera ordenada, es gestionar los recursos con una intensidad que permita garantizar la diversidad biológica, la productividad, la capacidad de regeneración, la vitalidad y la capacidad de cumplir las funciones ecológicas, económicas y sociales a corto, mediano y largo plazos.

El proceso de planeación del aprovechamiento de los recursos maderables del ejido Gómez Tepeteno se basó en estudios sobre las tasas de crecimiento de las principales especies comerciales. El resultado fue un horizonte de planeación de largo plazo que definió un turno promedio de crecimiento de 50 años, por lo que el aprovechamiento forestal se planeó en cinco ciclos de corta de 10 años que iniciaron en 1983. En 2013, a través de su delegación en el estado de Puebla, la Semarnat refrendó el aprovechamiento forestal maderable y la ejecución del cuarto ciclo de corta de su Programa de Manejo Forestal (PMF).

\section{Planeación del aprovechamiento forestal maderable}

$\mathrm{Al}$ inicio de cada año, las autoridades ejidales solicitan ante la Semarnat el número de remisiones forestales que utilizarán durante la anualidad de corta. Esto permite acreditar la procedencia legal en el transporte de la trocería del área de aprovechamiento al aserradero. Por otro lado, el presidente de la industria forestal del ejido se encarga de solicitar los reembarques que acreditarán el transporte de la madera aserrada a su destino final.

La organización ejidal para el aprovechamiento maderable de la EFC inicia con la elección de los ejidatarios que van a ocupar los puestos del Comité de Aprovechamiento (CA) (coordinador, jefe de monte y documentador). La elección se hace en la Asamblea Ejidal e inicia con la propuesta de ejidatarios para ocupar cada uno de los roles. Todos los asistentes a la asamblea votan a mano alzada por un candidato y son electos aquellos que tengan más votos.

Los entrevistados señalaron que los criterios que toman en cuenta para elegir a los miembros del CA son: la experiencia previa, la capacitación en el manejo de la forcípula electrónica y la inclusión de mujeres en las actividades en el bosque. Estos roles son asumidos de 
forma responsable por los ejidatarios seleccionados, ya que el Reglamento Interno Ejidal señala que los integrantes del CA no pueden renunciar sin previo análisis y autorización de la Asamblea Ejidal.

La actividad silvícola es realizada mayoritariamente por hombres, sin embargo, los integrantes de la Asamblea Ejidal mostraron interés para que las mujeres se involucren progresivamente en el aprovechamiento forestal, al emitir su voto a favor de una mujer, por primera vez en 2017, para que forme parte del CA en calidad de documentadora.

\section{Preparación de la fase de corte}

Existe mayor probabilidad de éxito en el cumplimento eficaz y eficiente de los objetivos comunes si se consideran los aspectos siguientes: la forma en que toda empresa organiza y distribuye el trabajo, el entorno, las capacidades de los individuos y de la organización, la tecnología, las innovaciones y los mecanismos de coordinación (López, Arias Montoya y Rave Arias, 2006). Estos elementos se observan claramente en la comunidad de estudio, ya que en el ejido Gómez Tepeteno, las autoridades ejidales, el CA y el presidente de la industria se reúnen con el personal que integrará las brigadas de trabajo antes de iniciar el aprovechamiento, con el propósito de brindar una capacitación inicial e intercambiar ideas sobre: logística, métodos de derribo, elaboración de trozas, minimización del impacto negativo sobre el capital natural, seguridad, uso de equipo de protección, limpieza de las áreas de corta y características de la madera en rollo para abastecer a la industria ejidal. Los aspectos que se enfatizan en la capacitación son: importancia del trabajo en equipo, comunicación y cumplimiento de normas para la generación de un buen ambiente laboral.

Después de la capacitación, el personal recibe su equipo de protección y se traslada a las áreas de corta junto con el jefe de monte. El coordinador del CA verifica que el material y los insumos estén disponibles para el buen funcionamiento del transporte forestal. Por último, la documentadora prepara la documentación (remisiones forestales, control de embarque de madera, libro de ventas y tablas de volumen), equipo de cómputo y forcípula electrónica.

Este proceso de capacitación ha permitido que la corta de árboles se desarrolle de manera coordinada, ordenada y segura porque todos los responsables de área y trabajadores conocen sus responsabilidades y saben las opciones de reacción ante cualquier eventualidad que suceda en el campo.

\section{Ejecución del corte}

La primera actividad en campo para el aprovechamiento maderable es la elaboración de la documentación de marqueo con base en los volúmenes autorizados en el PMF. En el mes de mayo de cada año, los asesores técnicos forestales del ejido, en presencia del Comisariado Ejidal y del Consejo de Vigilancia, se encargan de la selección y marqueo del arbolado a derribar.

En las zonas de aprovechamiento, el jefe de monte identifica los árboles marcados, con la finalidad de que el motosierrista principal dirija a las brigadas de derribo y se realice la corta del arbolado correctamente, con base en las necesidades de la industria.

Las tareas del corte del arbolado se presentan en la figura 1 , se incluyen las cuatro dimensiones que influyen en el proceso. Los elementos de la esfera social que interactúan son: capital social presente a través de relaciones de organización (Fukuyama, 2003), confianza, normas de reciprocidad, redes de intercambio y cumplimiento de normas formales e informales (Ostrom y Ahn, 2003) y capital humano expresado por habilidades, capacidades y experiencia de los ejidatarios en la ejecución de las tareas de derribo.

La esfera económica hace referencia a los beneficios producidos gracias al consumo de los bienes y servicios provenientes de los macizos forestales, los ingresos y empleos derivados del aprovechamiento forestal (FAO, 2014). En la dimensión político-institucional destaca la acción de las políticas públicas en la promoción del desarrollo de la EFC (Bray y Merino-Pérez, 2004). En este tenor, el ejido ha sido beneficiado por programas 
gubernamentales para adquirir o mejorar su capital natural, social, humano, físico y tecnológico.

La brigada de aprovechamiento se integra de la forma siguiente: un motosierrista principal, un ayudante de motosierrista, tres rameros, tres cableros y un gruyero (operador de la motogrúa). A excepción del motosierrista principal, que es un ejidatario con experiencia, los trabajadores de la brigada son civiles (personas avecindadas del ejido o de localidades aledañas).

La técnica de derribo que se utiliza es el derribo direccional para evitar perturbaciones al entorno y minimizar riesgos (Vargas-Larreta, 2013). Los ejidatarios han visto que el uso de esta técnica permite minimizar daños al arbolado residual, renuevo, cauces de agua y árboles secos que tienen una función importante como hábitat de flora y fauna (Secretaría de Economía [SE], 2008; Vargas-Larreta, 2013). El operador de la motosierra aplica los fundamentos anteriores para determinar el mejor sitio de caída del árbol (Fig. 2). Conforme avanza el derribo, los integrantes de la brigada de aprovechamiento se distribuyen el desrame, elaboración de brazuelo, leña y armado de estibas.

La actividad siguiente es el arrime a pie de brecha del fuste limpio para ser dimensionado. En esta actividad participan el gruyero que opera la motogrúa y tres cableros que se encargan del acomodo, enganche y desenganche del malacate de las trozas. Cabe mencionar que el arrime se realiza de forma semisuspendida para evitar afectaciones al suelo (Fig. 3).

El motosierrista principal dimensiona el fuste con base en diámetro, longitud y calidad de la materia prima a fin de obtener las mejores trozas, según sean de cortas y largas dimensiones. El ejido produce regularmente dos tipos de trozas: 1) Troza comercial de $2.55 \mathrm{~m}$ de largo y diámetro mayor a $31 \mathrm{~cm}$ y 2) Troza de cortas dimensiones de $1.27 \mathrm{~m}$ de largo con diámetro mayor a $15 \mathrm{~cm}$, sin embargo, las dimensiones de las trozas pueden variar de acuerdo con los pedidos de los clientes de la industria, especialmente porque el ejido está en el proceso de cambio a un esquema de integración de cadena de valor.
En la última tarea del corte, los rameros se encargan de picar las ramas y los residuos del aprovechamiento, que son distribuidos homogéneamente en curvas de nivel en el terreno, con el objetivo de evitar la erosión hídrica del suelo y disminuir el riesgo de incendios.

\section{La innovación de procesos en el abastecimiento forestal}

El ejido presenta interés en la innovación tecnológica en procesos de aprovechamiento y transformación. En 2016, el ejido ingresó una solicitud de apoyo ante la Conafor para Transferencia de Tecnología, con el fin de integrar el uso de la forcípula electrónica en la cubicación de madera en rollo y en la operación de un sistema automático de control contable y financiero, los cuales se ejecutan en la anualidad vigente (Fig. 4).

La introducción de la forcípula electrónica en las labores de registro y documentación implicó la participación de varios actores: integrantes del CA, personal de la Conafor, técnico especialista en el manejo de la forcípula electrónica, asesores técnicos del ejido y del promotor forestal comunitario. Este último lleva a cabo el acompañamiento y seguimiento de todas las actividades forestales del ejido.

El ejido Gómez Tepeteno es el primer ejido en México en usar la forcípula electrónica y el software especializado para el control contable en la documentación de madera en rollo, de acuerdo con la información proporcionada por la Coordinación General de Educación y Desarrollo Tecnológico de la Conafor por medio de la Plataforma Nacional de Transparencia del Gobierno Federal.

El técnico especialista en manejo de la forcípula electrónica capacitó teórica y prácticamente al jefe de monte, a la documentadora y al promotor forestal. También participó en el proceso de incorporación de la innovación al sistema de trabajo del ejido a nivel de campo, con el acompañamiento del CA en la documentación de la primera carga de madera en rollo. 


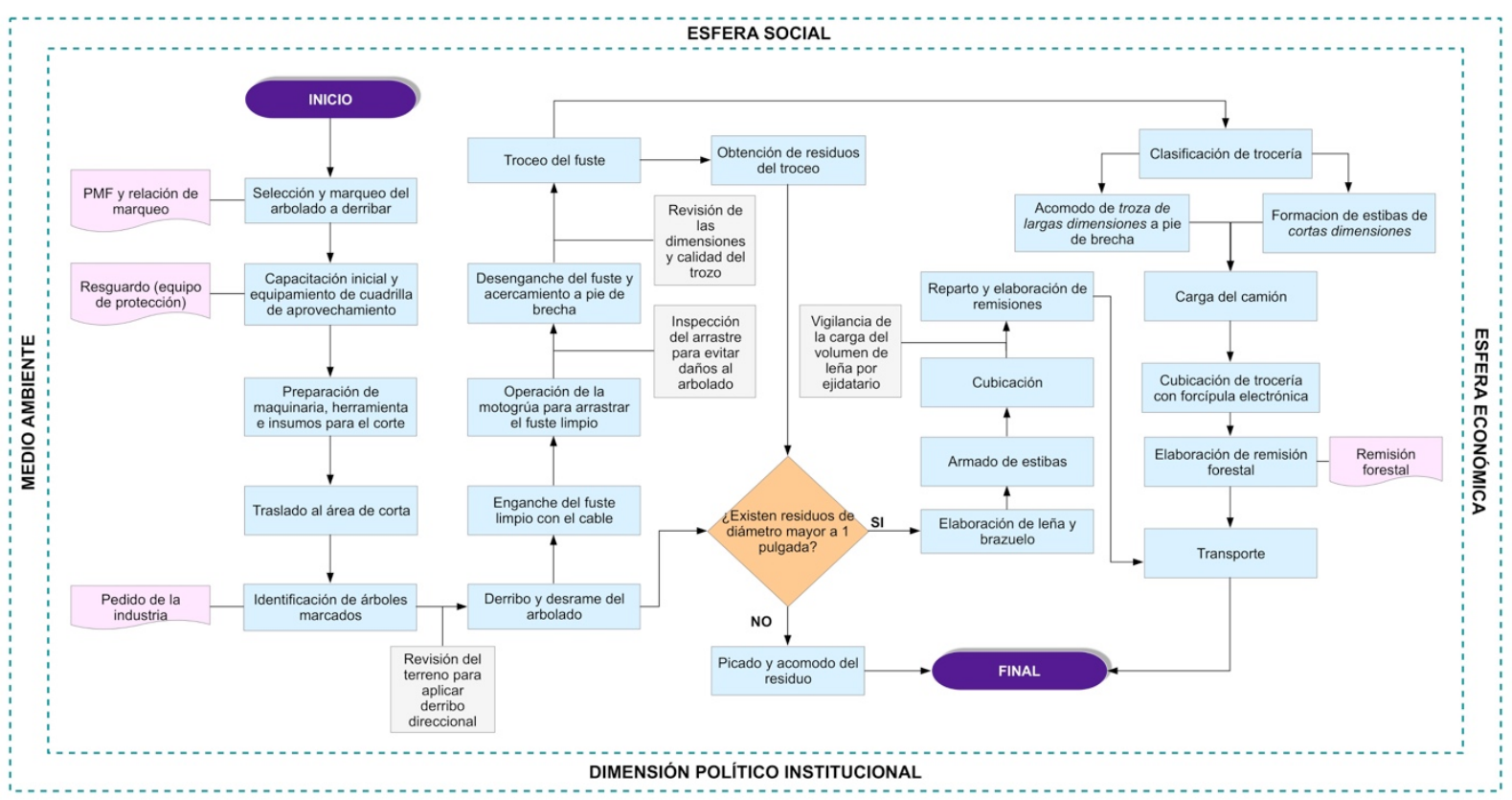

FIGURA 1. Diagrama de proceso de corte del arbolado.

Fuente: Elaboración propia.

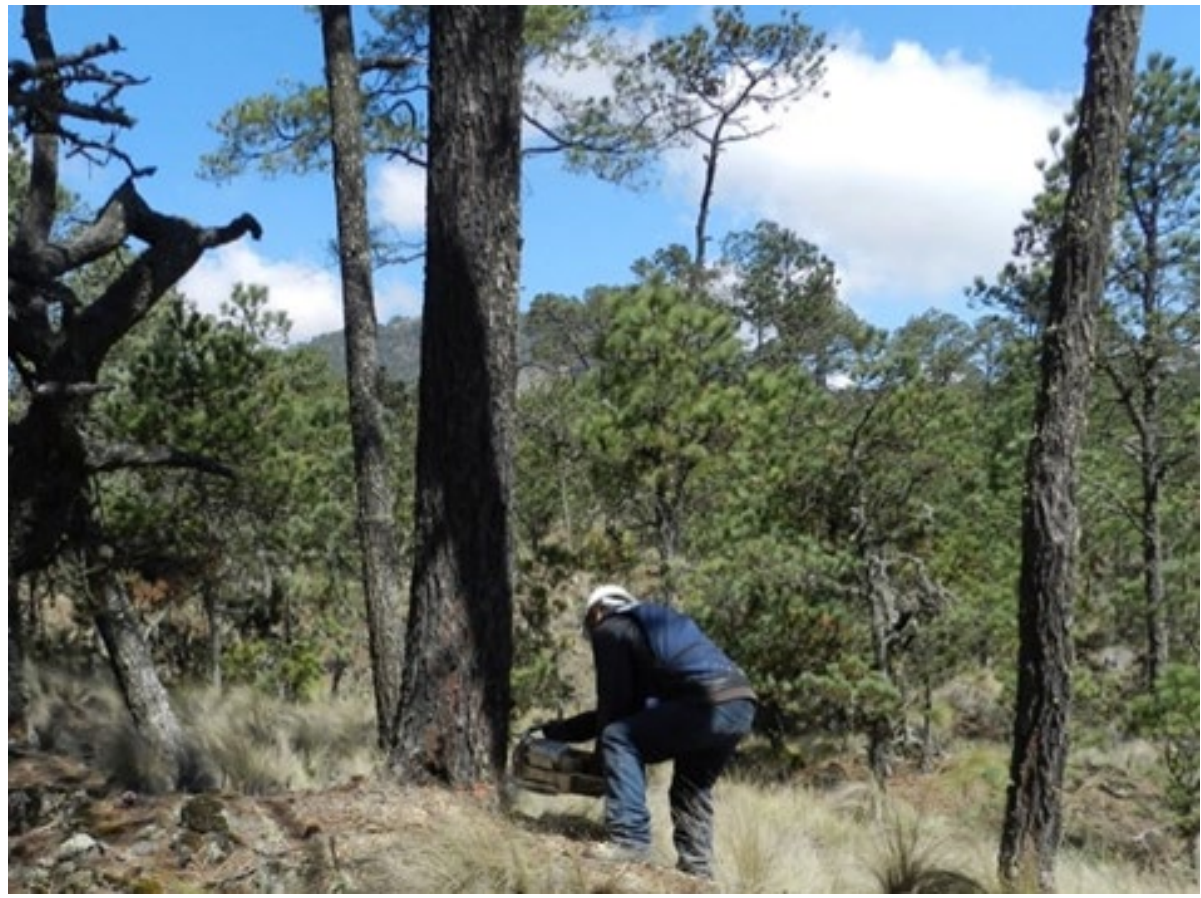

FIGURA 2. Aplicación del derribo direccional.

Fuente: Trabajo de campo (2017). 


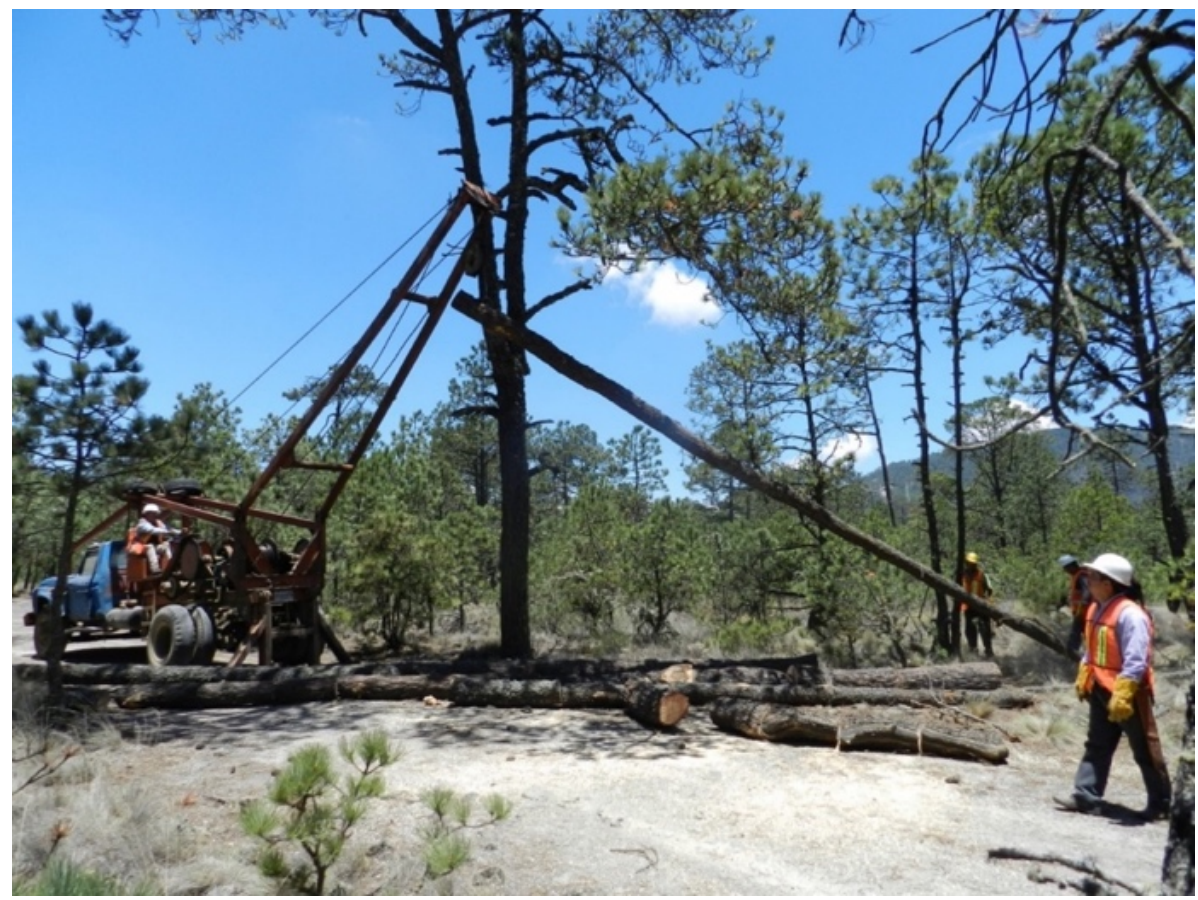

FIGURA 3. Arrime semisuspendido del fuste limpio.

Fuente: Trabajo de campo (2017).

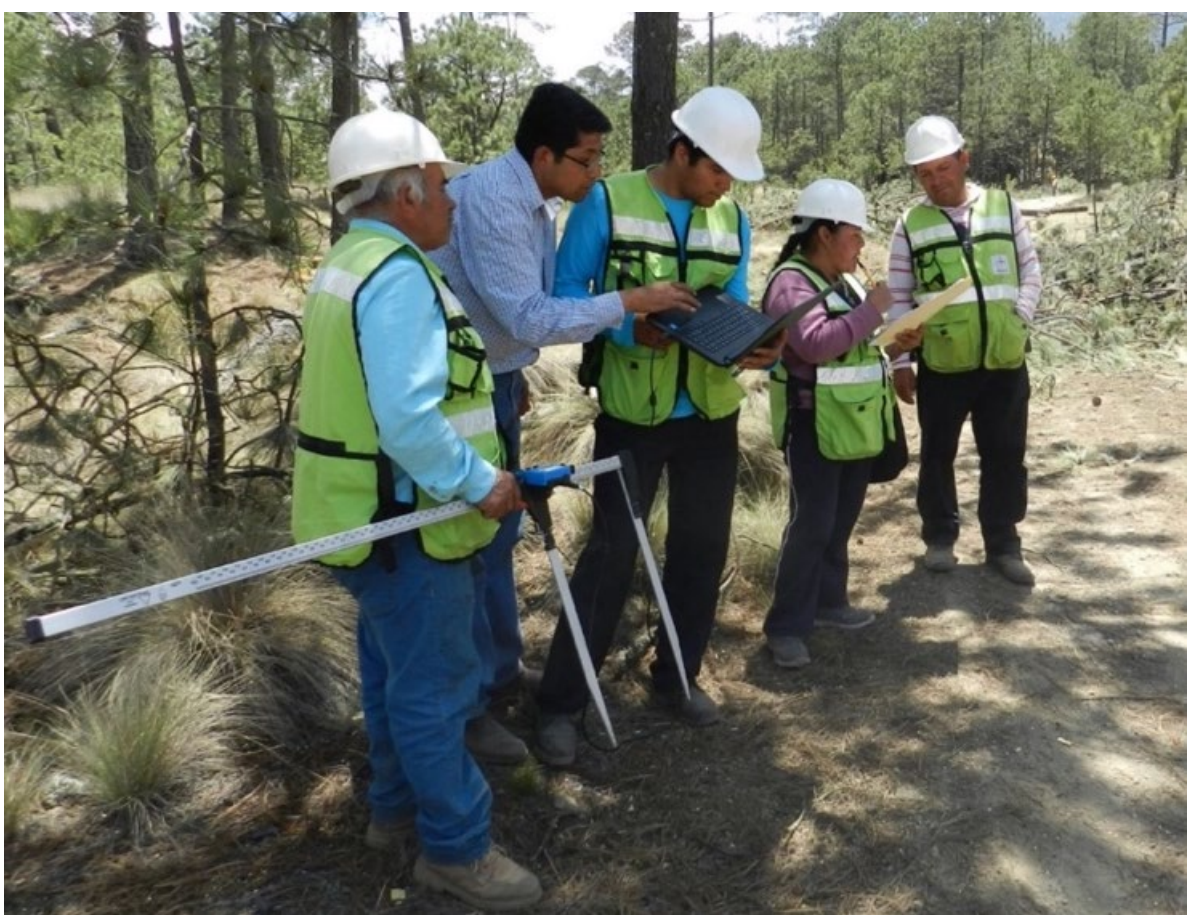

FIGURA 4. Forcípula electrónica Haglöf y sistema de control para la cubicación automática de madera en rollo.

Fuente: Trabajo de campo (2017). 
Los asesores técnicos forestales también capacitaron a la documentadora en el cálculo de los volúmenes de leña, brazuelo, trocería de cortas y largas dimensiones con uso del flexómetro. Se consideró importante capacitar a la documentadora en el método tradicional de cubicación de volúmenes para cuando no se pueda utilizar la forcípula electrónica por algún motivo. La documentadora también fue capacitada en el llenado de remisiones forestales porque ella tiene como función principal realizar la cubicación de volúmenes de madera en rollo cargada y la emisión de remisiones forestales para efectos del transporte de la trocería.

\section{Implementación de la innovación tecnológica en la documentación de la materia prima}

El jefe de monte avisa al personal responsable de transporte para que cargue el camión cuando considera que existe suficiente volumen de materia prima. Esta actividad aún se lleva a cabo de forma tradicional (Fig. 5). En ella participan el operador del camión y dos cargadores, los cuales usan el gancho maderero y la fuerza manual para subir y acomodar la trocería en el transporte forestal.

El jefe de monte y la documentadora utilizan la forcípula electrónica para calcular el volumen de la trocería que está cargada en el camión. Ellos miden los diámetros de la trocería (Fig. 6) y, conociendo la longitud, el software especializado hace el cálculo automático de la cubicación de la madera en rollo. La documentadora llena la remisión forestal con los volúmenes calculados para avalar la cantidad de materia prima transportada hacia el aserradero del ejido.

\section{Formación del capital humano para la operación de la industria forestal}

Los integrantes del ejido tuvieron inquietud en desarrollar su visión empresarial y capacidad de inversión a través de la creación de diversas empresas. En 1987, los ejidatarios incursionaron en la empresa del transporte de pasajeros, con la cual adquirieron experiencia en administración y manejo de recursos humanos, físicos y financieros. Las entrevistas muestran que, a partir del trabajo en la empresa de transporte, los ejidatarios lograron conciencia sobre los riesgos, el sacrificio que implica el manejo de una empresa y el tiempo requerido para obtener ganancias.

A partir de la experiencia empresarial anterior, un grupo de ejidatarios sometió a consideración de la Asamblea Ejidal la adquisición e instalación de un aserradero como un medio para combatir el intermediarismo, integrar el proceso de aprovechamiento forestal, darle valor agregado a la materia prima y generar empleos. En 2009, la Secretaría de Medio Ambiente y Recursos Naturales del Gobierno del Estado de Puebla materializó la decisión de la Asamblea Ejidal por medio de la entrega de un aserradero equipado con una desorilladora, un péndulo, tres tableteras y un taller de afilado. Un año más tarde, la misma dependencia otorgó una estufa de secado y una máquina para fabricar palos de escoba. Hasta el año 2017, todo el equipamiento y la maquinaria adquirida habían sido usados con excepción de la máquina para fabricar palos de escoba porque no han conseguido clientes, sin embargo, el Comité de la Industria tiene planes a corto plazo para iniciar la producción de palos de escoba.

En 2011, constituyeron la Sociedad de Producción Rural de Responsabilidad Ilimitada de Capital Variable denominada Grupo Ejidal GT, que tiene la responsabilidad del manejo y administración de la industria maderera, que incluye al aserradero y a la estufa. Al frente de la Sociedad se encuentra el Comité de la Industria integrado por Presidente, Secretario y Tesorero, cargos que son ocupados exclusivamente por ejidatarios y un auxiliar administrativo que es civil. Los puestos para la operación del aserradero son cubiertos por 24 trabajadores, de los cuales $92 \%$ son civiles (avecindados sin derechos agrarios) y $8 \%$ ejidatarios.

El establecimiento de la industria del ejido trajo consigo retos nuevos, entre ellos la formación de capital humano para ejecutar el proceso productivo de forma adecuada, con una productividad competitiva, con la seguridad necesaria y la coordinación de operaciones que permitan trabajar en grupo. El ejido Gómez Tepeteno ha reconocido el valor del conocimiento creado por las personas que integran la EFC como parte de su capital 


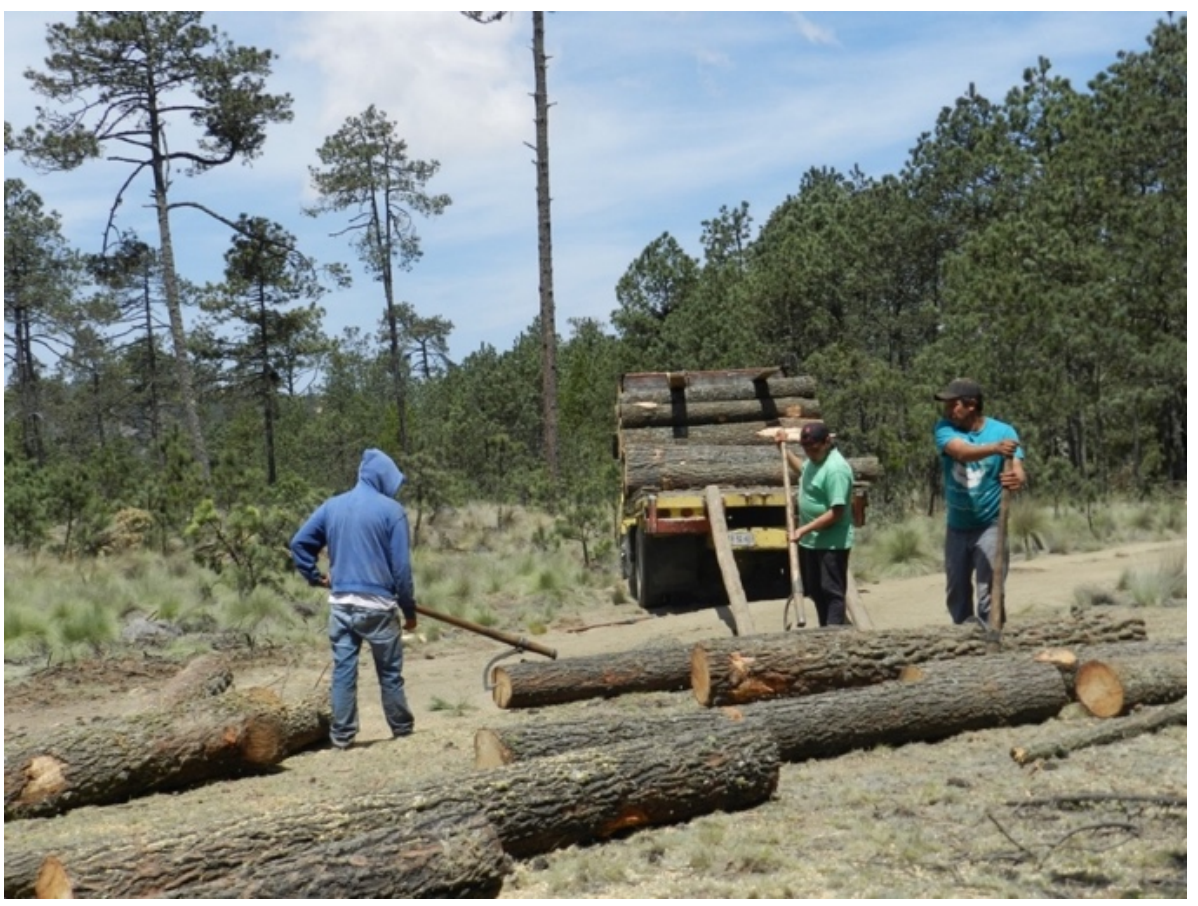

FIGURA 5. Carga de trocería de largas dimensiones.

Fuente: Trabajo de campo (2017).

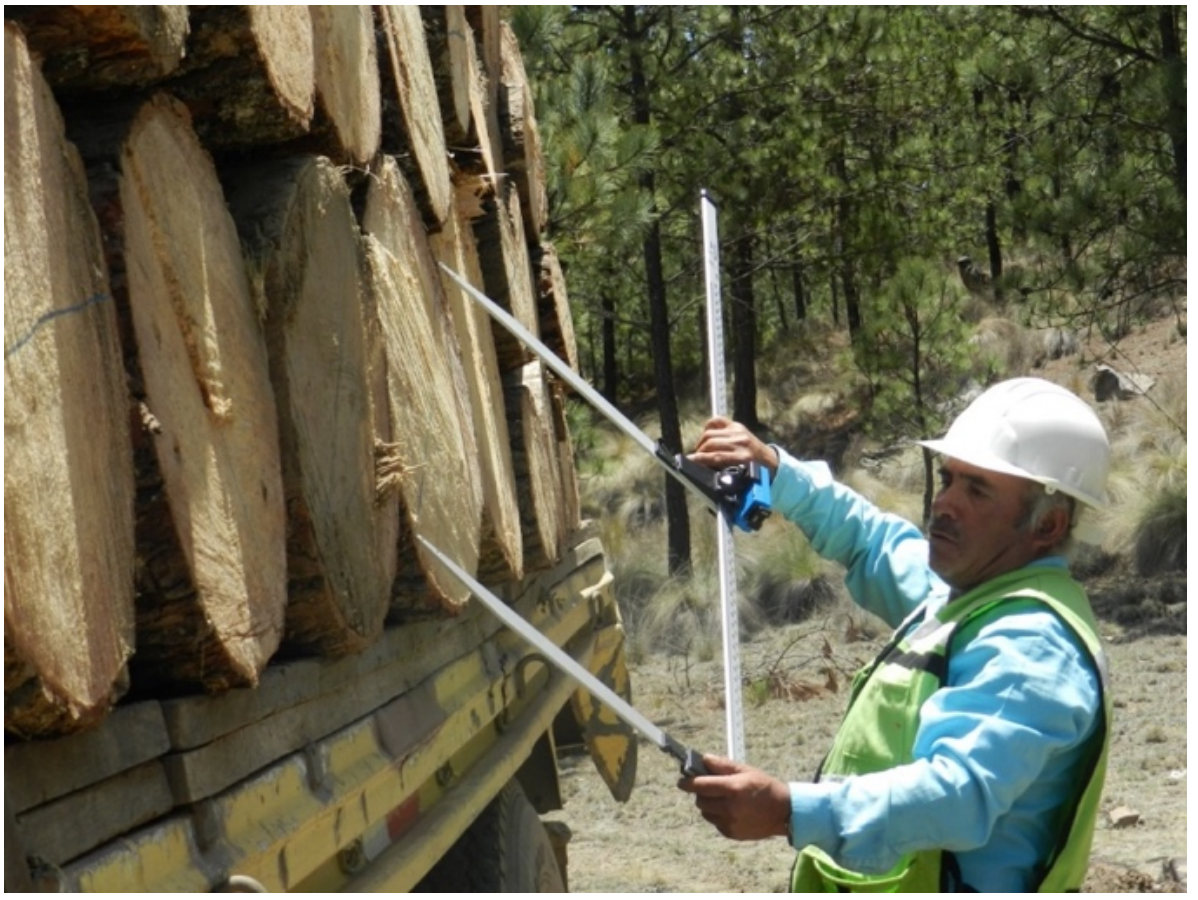

FIGURA 6. Adopción del uso de la forcípula electrónica Haglöf en la documentación de madera.

Fuente: Trabajo de campo (2017). 
humano que se expresa en sus habilidades, su capacidad de aprendizaje, competencias, capacitación y experiencia (López et al., 2006). El problema del capital humano es que es inherente a las personas, por lo que es necesario que la empresa establezca mecanismos que permitan valorar y mantener el conocimiento en la organización (López et al., 2006), a través de la capacitación de los trabajadores (ejidatarios y civiles) a partir de acciones directas de capacitación o derivado de la enseñanza de quienes tienen más experiencia.

Al inicio de la operación del aserradero, la totalidad del personal se formó a través de talleres, cursos, intercambio de experiencias y seminarios de comunidad a comunidad subsidiados por la Conafor. En la actualidad, se tiene la precaución de capacitar a cada trabajador nuevo del aserradero y a tener acompañamiento de parte del asesor técnico porque el ejido ha entendido que de eso depende la eficiencia productiva y la seguridad de los trabajadores.

$\mathrm{Al}$ aplicar las encuestas a civiles que trabajan en el aserradero, se obtuvo que $86 \%$ no contaba con ninguna experiencia previa en el área de trabajo que desempeña, 9\% expresó que tenía suficiente experiencia y 5\% indicó que tenía poca experiencia para realizar su actividad en la industria. El proceso de transmisión del conocimiento y desarrollo de habilidades en los civiles que trabajan en el aserradero se dio de diversas formas: la principal fue a través de la enseñanza de compañeros con mayor experiencia $(71 \%)$, seguido por capacitaciones organizadas por personal del ejido (24\%). Respecto a su expertise actual, una quinta parte $(19 \%)$ indicó que domina muy bien su actividad, más de la mitad (52\%) considera que ejecutan bien su trabajo y casi un tercio (29\%) piensa que lo hace regular.

\section{Transformación de la madera}

El proceso de transformación de la madera en el ejido Gómez Tepeteno se inicia cuando el jefe de patio solicita al operador del camión la remisión forestal y revisa información referente al número de trozas, descripción y volumen, así como datos del transporte empleado. En este punto de conexión entre el eslabón de corta y el eslabón de transformación, se establece una relación comercial como si fueran dos empresas en el mercado libre. El Comité de Aprovechamiento tiene que responsabilizarse de la cantidad de madera en rollo que entrega como si el aserradero ejidal fuera cualquier cliente y el Comité de la Industria tiene que revisar y constatar la cantidad y dimensiones de la materia prima que recibe como a cualquier otro proveedor.

La actividad siguiente es la descarga manual de materia prima. A continuación, el jefe de patio y el secretario miden las dimensiones de cada troza con flexómetro y apoyados por el comodín, registran los datos para calcular el volumen total de madera que ingresa al aserradero.

Cuando el camión ingresa al patio del aserradero puede pasar inmediatamente a la rampa de alimentación de la sierra principal cuando no hay materia prima, en caso contrario, se separa y clasifica en el patio considerando la calidad, tipo de troza y el padrón de producción, es decir, el número de piezas de dimensiones específicas que se deben producir con base en el requerimiento de los clientes de madera aserrada (Rainforest Alliance, Reforestamos México y Consejo Civil Mexicano, 2010).

En este punto es necesario señalar que el ejido está evolucionando de una cadena productiva a trabajar bajo el modelo de cadena de valor. De acuerdo con Fideicomisos Instituidos en Relación a la Agricultura (FIRA) una red o cadena de valor es un modelo que busca una mejor vinculación de la producción primaria con el mercado, a través de la organización de agentes económicos para producir y llevar un bien particular al consumidor (FIRA, 2014). El concepto de cadena de valor usado en el presente artículo se tomará como equivalente del concepto de red de valor, mencionado por FIRA (2014). Desde este enfoque, el consumidor define qué productos se deben producir, las características de los productos, lugar de compra, temporada de compra, etc. Por tanto, la cadena de valor se integra para satisfacer la demanda del cliente de forma organizada, competitiva y buscando que los beneficios se reflejen en todos los agentes que la integran. Cabe señalar que el ejido recibió apoyo del Programa de Naciones Unidas para el Desarrollo con el propósito de conseguir 
clientes para su madera aserrada. En la actualidad, casi toda su madera aserrada es vendida a clientes con los que se establecen contratos de compra-venta de productos específicos (Fig. 7). La madera que el ejido comercializa en el mercado libre es mínima.

Los clientes principales de la industria de Gómez Tepeteno demandan tablas, tablones y habilitados para tarima con dimensiones específicas. Otros productos que se producen en menor volumen son: cuartón, polín, viga, habilitado para caja, costera y cuadrados para palo de escoba.

La figura 8 ilustra el flujo que siguen la mayor parte de los productos del aserradero desde la recepción de la trocería hasta que llega al consumidor final. Es importante resaltar que el jefe del aserradero es el responsable de coordinar y supervisar las actividades con el objetivo de cumplir los pedidos en tiempo y forma.

La última actividad de la transformación de la madera consiste en el secado, fase en la que el ejido también ha innovado. El secado de las tablas constituye una fase crítica de la industrialización ya que dependiendo del método utilizado será el tiempo para obtener la madera para su comercialización y también determina la calidad del producto final (Solís-Rodríguez, Cerón-Cardeña y González-Ahumada, 2003). El secado se puede realizar de tres formas: al aire libre, mediante deshumidificación o bomba de calor y por medio de estufas (HernándezPalafox, 2014). En el ejido Gómez Tepeteno, el secado de la madera se realiza por dos métodos: al aire libre y con estufa.

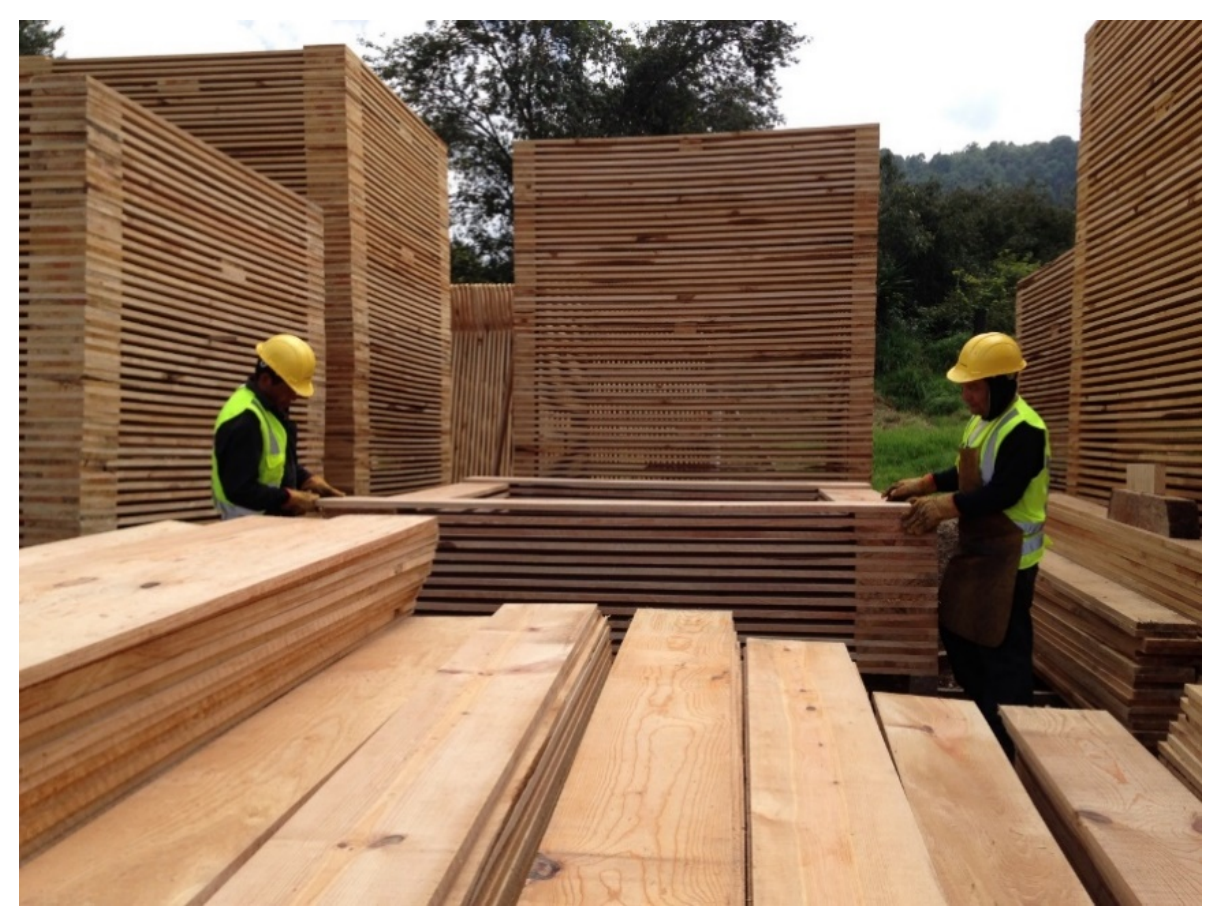

FIGURA 7. Formación de estibas con madera aserrada de dimensiones comerciales.

Fuente: Trabajo de campo (2017). 


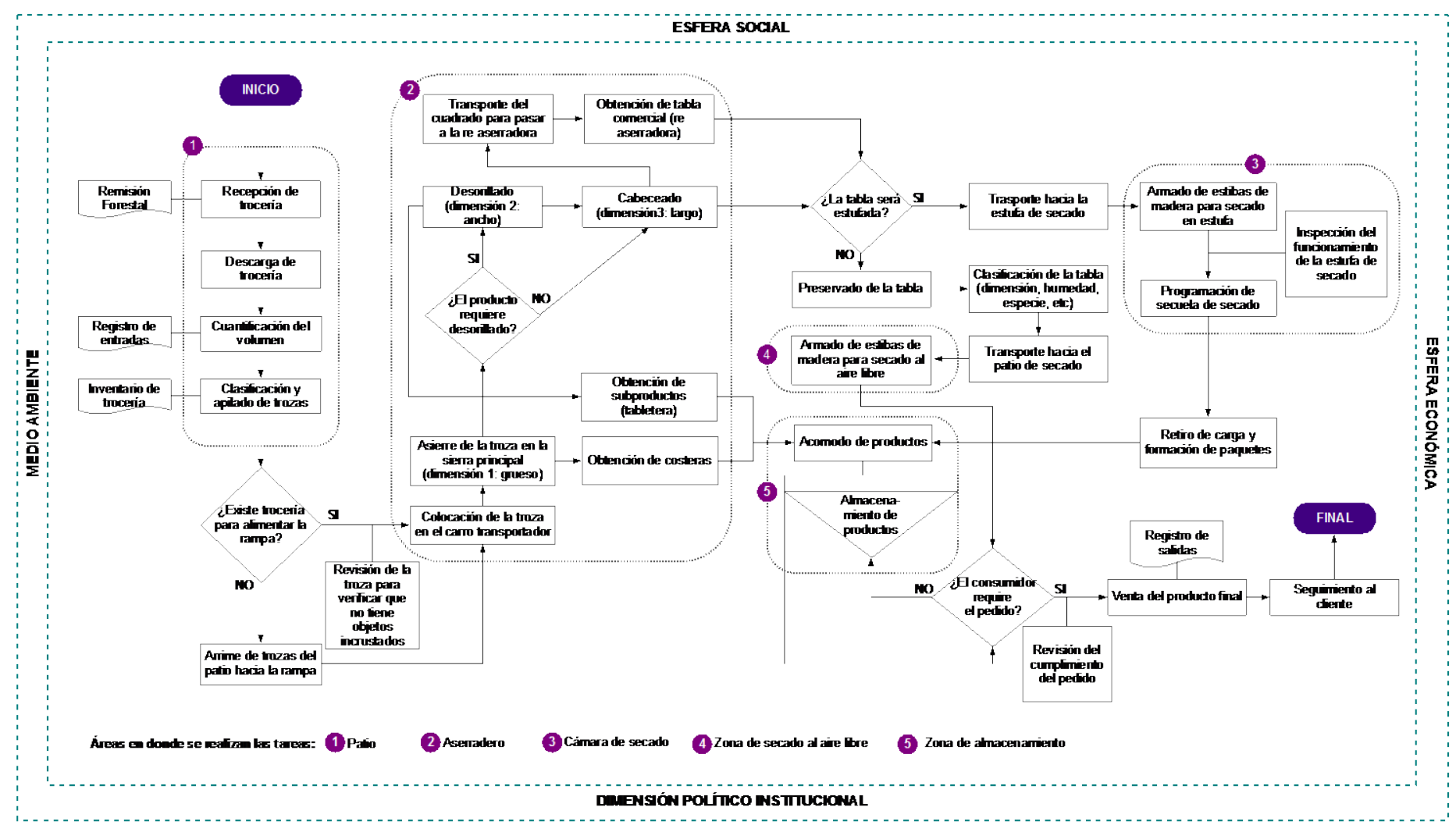

FIGURA 8. Proceso de producción de la industria forestal del Ejido Gómez Tepeteno.

Fuente: Elaboración propia.

El secado al aire libre requiere de una inversión mínima, pero tiene las desventajas de ser un método lento y su efectividad depende de las condiciones climáticas del lugar y de las características físico mecánicas de las tablas (Hernández-Palafox, 2014). Además puede producir variaciones importantes en la calidad de la madera, aun dentro de cada lote de tablas, por la variación de los elementos del clima. En cambio, el secado artificial usando una estufa para madera requiere inversión inicial alta (SolísRodríguez et al., 2003), pero tiene varias ventajas: llegar al punto de secado requerido por el cliente, es un método más rápido respecto al anterior, elimina el peligro del manchado o pudrición de la madera y genera una calidad uniforme en todo el lote cuando hay un buen manejo del estufado (Hernández-Palafox, 2014).

La cuenca de abasto a la cual pertenece el ejido Gómez Tepeteno se conforma por siete municipios considerados de elevado potencial forestal: Chignautla, Cuyoaco,
Tlatlauquitepec, Xochiapulco, Zacapoaxtla, Zaragoza y Zautla. En la cuenca existen 24 industrias forestales en actividad, de las cuales 12 se localizan en el municipio de Tlatlauquitepec (Ingeniería Agroforestal y Ambiental del Bosque Mesófilo S.C. [Inafam], 2015). Inafam (2015) señala que de las cinco estufas de secado instaladas en la cuenca de abasto, tres están operando y solo la del Grupo Ejidal GT del ejido de Gómez Tepeteno además de secar su producción, brinda el servicio de maquila de secado, posicionando a la industria de este ejido como referente de la cuenca de abasto. Este es un punto estratégico que el ejido de Gómez Tepeteno debe aprovechar para impulsar el resto de sus actividades del aprovechamiento maderable. Por último, la figura 9 presenta la visión conjunta de la cadena de valor del ejido, partiendo del elemento organizacional hasta la transformación de la materia prima forestal para su comercialización. 


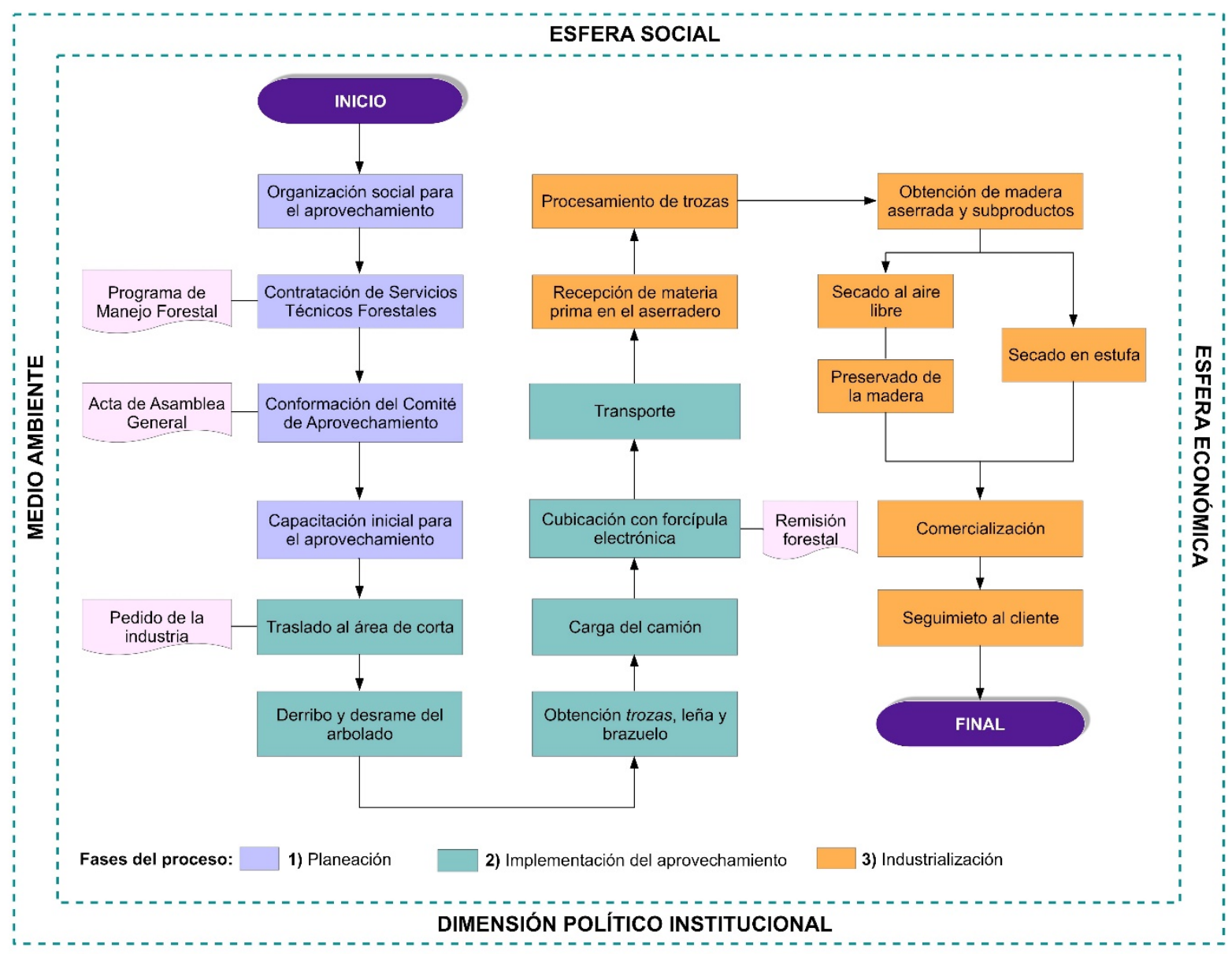

FIGURA 9. Diagrama general de la integración de la cadena de valor del ejido Gómez Tepeteno.

Fuente: Elaboración propia.

\section{CONCLUSIONES}

La Asamblea Ejidal es la máxima expresión del capital social a través de la cual se organizan todas las actividades productivas del ejido. Es un órgano que tiene la mayor jerarquía en las decisiones que giran en torno al aprovechamiento de los recursos forestales, pero puede delegar algunas funciones y decisiones en algunos órganos ejidales como son: el Comisariado Ejidal, el Consejo de Vigilancia, el Comité de Aprovechamiento, el Comité de la Industria, entre otros.

La planeación de las actividades y los procesos de capacitación previos y durante los ciclos de corta e industrialización han permitido la formación de capital humano para que los procesos productivos se desarrollen de forma eficiente y segura, teniendo especial cuidado en la conservación del capital natural.

El ejido ha generado sistemas permanentes de innovación tecnológica en los procesos de aprovechamiento y transformación que implican inversión y capitalización en el corto, mediano y largo plazos. Dos ejemplos importantes de la innovación son: el uso de la forcípula en la fase de abastecimiento y uso de estufa de secado de madera aserrada. El uso de la forcípula electrónica ha permitido reducir el tiempo y hacer la cubicación de madera en rollo de manera más precisa para su documentación. La estufa de secado de la madera aserrada ha permitido reducir los 
tiempos de secado, ampliar la gama de servicios que ofrece la industria, generar productos de alta calidad uniforme y posicionar a la industria del ejido como referente de la cuenca de abasto a la cual pertenece.

El ejido Gómez Tepeteno ha fortalecido su capital social a partir de una organización interna que les ha permitido lograr un buen funcionamiento de sus procesos productivos. El capital social también se ha fortalecido con los procesos de integración a redes de apoyo con instituciones estatales, nacionales e internacionales a través de financiamiento, equipamiento, capacitación, acompañamiento y acceso a los mercados.

Finalmente, el modelo de aprovechamiento del ejido Gómez Tepeteno ha logrado avances importantes pero está en una fase de consolidación pasando de una cadena de producción a una cadena de valor, con lo cual se crea una relación superior entre productor y cliente.

\section{REFERENCIAS}

Aguirre-Calderón, O. A. (2015). Manejo forestal en el siglo XX. Madera y Bosques, 21(Núm. Esp.), 17-28. doi: http://dx.doi.org/10.21829/myb/2015.210423

Boisier, S. (2003). ¿Y si el desarrollo fuera una emergencia sistémica?. Cuadernos de Administración, 19(29), 47-80. doi: 10.25100/cdea.v19i29.123

Bovet, D., \& Joseph, M. (2000). V alue nets: braking the supply chain to unlock hidden profits. Estados Unidos: Mercer Management Consulting.

Bray. D. B., Merino-Pérez, L., Negreros-Castillo, P., SeguraWarnholtz, G., Torres-Rojo, J. M., \& H. Vester. (2003). Mexico's community-managed forests as a global model for sustainable landscapes. Conservation Biology, 17(3), 672677. doi:10.1046/j.1523-1739.2003.01639.x

Bray, D. B., \& Merino-Pérez, L. (2004). La experiencia de las comunidades forestales en México: Veinticinco años de silvicultura y construcción de empresas forestales comunitarias. México, Distrito Federal: Secretaría de Medio Ambiente y Recursos Naturales, Instituto Nacional de Ecología, Consejo Civil Mexicano para la Silvicultura Sostenible y Fundación Ford.

Chao, S. (2012). Pueblos de los bosques: cifras mundiales. Reino Unido: Forest Peoples Programme.
Chapela, F. (2007). El manejo forestal comunitario indígena en la Sierra de Juárez, Oaxaca. En D. Bray, L. Merino-Pérez, \& D. Barry (Eds), Los bosques comunitarios en México (pp.123145). México: INE-Semarnat.

Chapela, G. (2012). Problemas y oportunidades en el mercado para las empresas sociales forestales en México. México: Consejo Civil Mexicano para la Silvicultura Sostenible A.C., Universidad Autónoma Chapingo, Agencia de los Estados Unidos para el Desarrollo Internacional.

Fideicomisos Instituidos en Relación a la Agricultura [FIRA] (2014). Mapeo de redes de agronegocios. Boletín Informativo Nueva Época, 21, 9-79.

Fukuyama, F. (2003). Capital social y desarrollo: la agenda venidera. En R. Atria, M. Siles, I. Arriagada, L. J. Robison, \& S. Whiteford (Comps.), Capital social y reducción de la pobreza en América Latina y el Caribe, en busca de un nuevo paradigma (pp. 33-48). Chile: Comisión Económica para América Latina y El Caribe/Michigan State University.

Gasca-Zamora, J. (2014). Gobernanza y gestión comunitaria de recursos naturales en la Sierra Norte de Oaxaca. Región y Sociedad, 26(60), 89-120. Recuperado de

http:/ / www.scielo.org.mx/scielo.php?script=sci_arttext\&pid=S 187039252014000300004\&lng=es\&tlng=es.

H. Congreso de la Unión. (1986). Ley Forestal. Publicada en DOF del 31 de mayo de 1986. México. 13 p.

H. Congreso de la Unión. (2003). Ley General de Desarrollo Forestal Sustentable. Publicada en el DOF del 25 de febrero de 2003. Última reforma publicada DOF 24-01-2017. México. 82 p.

Hernández-Palafox, E. (2014). Material de construcción para hornos de secado de madera. Cientifica, 18(4), 141-145.

Ingeniería Agroforestal y Ambiental del Bosque Mesófilo S.C. [Inafam]. (2015). Estudio de cuenca de abasto forestal Teziutlán Puebla México. México: Secretaría de Medio Ambiente y Recursos Naturales, Comisión Nacional Forestal.

López D, M. E., Arias-Montoya, L., \& Rave-Arias, S. N. (2006). Las organizaciones y la evolución administrativa. Scientia Et Technica, XII(31), 147-152.

López-Barrera, F., Velázquez, A., \& Merino-Pérez, L. (2010). Explorando los determinantes del buen manejo forestal comunitario. Interciencia, 35(8), 560-567.

Merino-Pérez, L., \& Segura-Warnholtz, G. (2007). Las políticas forestales y de conservación y sus impactos en las comunidades forestales en México. En D. Bray, L. Merino- 
Pérez, \& D. Barry (Eds), Los bosques comunitarios en México (pp. 77-98). México: INE-Semarnat.

Organización de las Naciones Unidas para la Alimentación y la Agricultura [FAO]. (2004). Estudio de tendencias y perspectivas del sector forestal en América Latina al año 2020. Informe Nacional México. Recuperado de http://www.fao.org/docrep/006/j2215s/j2215s00.htm\# TopOfPage.

Organización de las Naciones Unidas para la Alimentación y la Agricultura [FAO]. (2010). Evaluación de los recursos forestales mundiales 2010 (Informe principal 163), Roma, Italia: FAO.

Organización de las Naciones Unidas para la Alimentación y la Agricultura [FAO]. (2014). El estado de los bosques del mundo. Potenciar los beneficios socioeconómicos de los bosques. Roma, Italia: FAO.

Ostrom, E., \& Ahn, T. K. (2003). Una perspectiva del capital social desde las ciencias sociales: capital social y acción colectiva. Revista Mexicana de Sociología, 65(1), 155-233. Recuperado de http:/ / www.scielo.org.mx/scielo.php?script=sci_arttext\& pid $=$ S0188-25032003000100005\&lng=es\&tlng=es.

Rainforest Alliance, Reforestamos México y Consejo Civil Mexicano para la Silvicultura Sostenible. (2010). Manual de buenas prácticas en aserraderos de comunidades forestales. México: Rainforest Alliance, Reforestamos México y el Consejo Civil Mexicano para la Silvicultura Sostenible.

Schultz, T. W. (1972). Capital humano: aspectos de política y oportunidades de investigación en Oficina Nacional de Investigación Económica. Investigación Económica: retrospectiva y prospectiva, $6,1-84$.

Secretaría de Economía [SE] (2008). Declaratoria de vigencia de la Norma Mexicana NMX-AA-143-SCFI-2008, para la certificación del manejo sustentable de los bosques. México, D.F.: SE.

Secretaría de Medio Ambiente y Recursos Naturales [Semarnat] (2012). Inventario Nacional Forestal y de Suelos México. Informe de resultados 2004-2009. Jalisco, México: Secretaría de Medio Ambiente y Recursos Naturales. Coordinación General de Planeación e Información a través de la Gerencia de Inventario Forestal y Geomántica.
Secretaría de Medio Ambiente y Recursos Naturales [Semarnat]. (2014). Programa Nacional Forestal 2014-2018. México: Secretaria de Medio Ambiente y Recursos Naturales. Diario Oficial de la Federación. 28 de abril de 2014.

Solís-Rodríguez, L. E., Cerón-Cardeña, M. A., \& GonzálezAhumada, I. (2003). Diseño y operación de una estufa solar para secar madera. Ingeniería, 2003, 35-48.

Torres-Rojo, J. M., Guevara-Sanginés, A., \& Bray, D. B. (2007). La economía de la administración del manejo comunitario forestal en México: Un estudio de caso en el Balcón, Tecpan, Guerrero. En D. B. Bray, L. Merino-Pérez, \& D. Barry (Eds), Los bosques comunitarios en México (pp. 343-375). México: INE-Semarnat.

Vargas-Larreta, B. (2013). Manual de mejores prácticas de manejo forestal para la conservación de la biodiversidad en ecosistemas templados de la región norte de México. México: Programa de las Naciones Unidas para el Desarrollo, Secretaría de Medio Ambiente y Recursos Naturales, Comisión Nacional Forestal y Rainforest Alliance.

Manuscrito recibido el 21 de octubre de 2017

Aceptado el 14 de marzo de 2018

Publicado el 1 de noviembre de 2018

Este documento se debe citar como:

Sánchez-Vidaña, D. L., Valtierra-Pacheco, E., González-Guillén, M. J., \& León-Merino, A. (2018). Capital humano e innovación en el proceso de integración del aprovechamiento forestal maderable en el ejido Gómez Tepeteno, Tlatlauquitepec, Puebla. Madera y Bosques, 24(2), e2431654. doi: 10.21829/myb.2018.2431654

Madera y Bosques por Instituto de Ecología, A.C. se distribuye bajo una Licencia Creative Commons Atribución-NoComercialCompartirlgual 4.0 Internacional. 